\title{
Identification of organic contaminants in disposable aluminium foil containers
}

\author{
F. TATEO \& M. BONONI \\ University of Milan, Di. Pro. Ve., Via Celoria, 2, Milan, I-20133, Italy
}

(Received 21 December 2005; revised 8 May 2006; accepted 13 May 2006)

\begin{abstract}
A series of disposable aluminium foil containers designed for cooking and packaging foods and available on the Italian market were investigated. Using different analytical techniques (near infrared (NIR), High Resolution Gas Chromatography (HRGC), gas chromatography/mass spectrometry (GC/MS)), organic substances used as lubricants in the manufacturing of the containers were detected on the aluminium layer in contact with the food. These contaminants were not mineral hydrocarbon fractions used for the shaping of can lids and bodies, but had an organic structure identifiable as esters derived by synthesis between fatty acids C8 and C10 with glycerine, and between C16 and C18 with butyl alcohol. Mixtures of higher esters were also identified as contaminants. Twelve samples were analysed, and the total amount of contaminant substances was found to range from 900 to $1200 \mu \mathrm{g} \mathrm{dm}^{-2}$. It is recommended, for safety and compliance with European Community (EC) Regulation No. 1935/2004 (27 October 2004), that the use of aluminium foil containers be subject to appropriate regulation concerning substances adopted for technological purposes.
\end{abstract}

Keywords: Aluminium foil containers, migration, lubricants, taint

\section{Introduction}

In compliance with European Commission (EC) Regulation No. 1935/2004 (European Community 2004), materials and articles intended to come into direct or indirect contact with food must be sufficiently inert to prevent substances from being transferred to food in quantities large enough to do the following:

- Endanger human health.

- Bring about unacceptable changes in the composition of the food.

- Cause a deterioration of sensory characteristics.

Point 10 of the Introduction section of this Regulation states that it is necessary to lay down various types of restrictions and conditions for the use of the materials and articles covered by this Regulation and the substances used in their manufacture'. At present, no list of substances that may potentially migrate from aluminium containers to food are included in any EC Directive. Moreover, no legally allowable migration limits are available for substances of known or unknown origin. Consequently, no tolerances are provided for by law for any residual substance derived from the technological treatment of aluminium surfaces. In anticipation of impending relevant legal decisions on this topic, the present study identifies technologically relevant substances used in the production of aluminium items. The chemical nature of these substances and related health information are urgent issues that have not yet been solved.

In the last 10 years, data have been published on aluminium migration due to contact with acid food (Gramiccioni et al. 1996). Results show that migration is a function of temperature, contact duration and food composition. Checks on aluminium migration (Feliciani et al. 2005) have been carried out following Commission Directive EEC/90/128, dated 23 February 1990 (European Commission 1990), relating to plastic materials and articles intended to come in contact with foodstuffs. These results showed that migration data in olive oil at $175^{\circ} \mathrm{C}$ 
for $2 \mathrm{~h}$ or $3 \%$ acetic acid at $100^{\circ} \mathrm{C}$ for $0.5 \mathrm{~h}$ was lower than the limit of detection. Based on these migration values, it has been assumed that there were no consumer health risks under proper, ordinary use of aluminium foil containers. Previous studies have focused on food contamination due to the migration of mineral hydrocarbons deriving from press lubricants present in cans supplied by industry for food and beverages (Jickells et al. 1994), and food contamination by identifying mineral oils and release agents by liquid chromatography/gas chromatography (LC-GC) (Grob et al. 1991a, b, 1992). Research in this area was extended to other organic matter that may be found at the bottom of aluminium foil containers due to the observation that not only is metal waxing with mineral hydrocarbons essential as a lubricant during moulding processes, but also other materials that were used as release agents. Unfortunately, no information on the chemical nature of the latter lubricants is supplied by manufacturers.

The present study presents preliminary experimental results that were obtained by investigating a series of disposable aluminium foil containers designed for food cooking and available on the Italian market, with the aim of verifying whether some substances - designated as contaminants in the present research - were present on the aluminium layer in contact with food. The aim of the work was to use analytical methods to identify the most common organic substances such as triglycerides and some other esters of fatty acids that may be used as lubricants to facilitate moulding of aluminium foil containers and remain as contaminants on the inner surface.

\section{Materials and methods}

Chemicals

Hexane GC analytical grade $\geq 98.0 \%$ purity was obtained from Merck (Darmstadt, Germany). The C21 standard of glycerol trienanthate $\geq 99.0 \%$ purity was purchased from Fluka (Milan, Italy) and was used as internal standard. Potassium bromide $>99 \%$ purity, Fourier Transformate-Infra Red (FT-IR) grade, was obtained from Sigma Aldrich (Milan, Italy). Standard butter fat used as a triglyceride standard certified reference material BCR-CRM 519, which was supplied from the Institute for Reference Material and Measurements (Geel, Belgium).

\section{Samples}

Twelve samples of aluminium foil containers were collected and classified with code numbers (from
$\mathrm{P} 84 / 1$ to $\mathrm{P} 84 / 12$ ) to grant privacy in the study, according to procedure PR 06 included in the Quality Management System adopted by Analytical Research Laboratories, Food and Environment, University of Milan, Italy (Quality Management System 2004). Samples were purchased from Italian supermarkets in Northern Italy and collected in order to examine the most well-known and best brands of containers.

\section{Sample preparation}

For FT-near infrared (NIR) analysis, approximately $5 \mathrm{ml}$ of hexane were used to wash the inner bottom of aluminium foil containers by shaking moderately, and the extract was transferred to a Petri dish. In parallel, the same amount of pure solvent (control) was placed into another Petri dish. The solvent from both dishes was allowed to evaporate at room temperature by placing both dishes in a vacuum dryer. For analysis, the same residue was used as that obtained upon evaporation, as described above. To prepare the alkali halide disc, the dried matter was mixed with potassium bromide (transmission method).

GC identification and characterization of both triglycerides and other esters of fatty acids (i.e. butyl palmitate and butyl stearate) were made on hexane extract, produced as described above. Briefly, $10 \mathrm{ml}$ of hexane were used to wash the inner bottom of aluminium foil containers by shaking moderately and reduced to a volume of $2 \mathrm{ml}$ in a vacuum dryer. This solution was injected for GC and gas chromatography-mass spectrometry (GC-MS) analyses.

For GC quantitative evaluation, $0.1 \mathrm{ml}$ of internal standard was added to the hexane extract (three times $\times 5 \mathrm{ml}$ ), which had been previously transferred to a graduated screw-cap glass vial. After concentration to $2 \mathrm{ml}$ at room temperature under vacuum, the solution was injected for GC analysis.

\section{Equipment}

A NIRFlex N-500 spectrometer equipped with Fiber Optic Solids (Büchi Labortechnik AG, Switzerland) was used. Analysis in transflectance was performed using a stainless steel cover (Transflectance Cover, TFKO). Büchi Calibration for chemical parameters was developed using NIRCAL 5.0 Chemometric software (Labortechnik AG, Switzerland).

FT-IR measurements were carried out using an Avatar 370 (Thermo Nicolet Corp., Madison, WI, USA). EZ OMNIC software was used to collect spectra.

A Carlo Erba gas chromatograph 5160 Mega Series coupled to a flame ionization detector (Milan, Italy), operated with hydrogen as the carrier gas at $20 \mathrm{kPa}$ head pressure, was used for GC analysis. 
A Petrocol $^{\text {TM }}$ EX2887 Supelco column, $5 \mathrm{~m}$ length $\times 0.53 \mathrm{~mm}$ i.d., $0.1 \mu \mathrm{m}$ film phase (Milan, Italy), was employed for the separation procedure. The following conditions were set for GC analysis: injector block at $50^{\circ} \mathrm{C}$; injection of $0.4 \mu \mathrm{l}$ was made on column mode; Flame Ionization Detector (FID) temperature held at $340^{\circ} \mathrm{C}$. The column was held at $150^{\circ} \mathrm{C}$ for $1 \mathrm{~min}$ after injection, and programmed to rise $10^{\circ} \mathrm{C} \mathrm{min}^{-1}$ to $200^{\circ} \mathrm{C}$, then $5^{\circ} \mathrm{C} \mathrm{min}^{-1}$ to $320^{\circ} \mathrm{C}$, and held at this temperature for $15 \mathrm{~min}$.

GC/MS analyses were performed using a Shimadzu 2010 gas chromatograph coupled to a Shimadzu QP-2010 MSD quadrupole mass spectrometer. Suitable separation of analytes was achieved using an Equity $^{\mathrm{TM}_{-}}$-5 [poly (5\% diphenyl/95\% dimethylsiloxane] capillary column, $30 \mathrm{~m}$ length $\times$ $0.25 \mathrm{~mm}$ i.d., $0.25 \mu \mathrm{m}$ phase (Supelco, Milan, Italy). The injection volume was $2 \mu \mathrm{l}$ with split ratio of 1:5. Helium was employed as the carrier gas with a column flow rate of $1.0 \mathrm{ml} \mathrm{min}{ }^{-1}$. Initial oven temperature was $200^{\circ} \mathrm{C}(1 \mathrm{~min})$, then $4^{\circ} \mathrm{C} \min ^{-1}$ to $280^{\circ} \mathrm{C}$. The mass spectrometer scanned from 40 to $500 \mathrm{~m} / \mathrm{z}$; ion source was set at $200^{\circ} \mathrm{C}$; injector temperature was $250^{\circ} \mathrm{C}$; detector temperature was $250^{\circ} \mathrm{C}$; capillary direct interface temperature was $250^{\circ} \mathrm{C}$. Spectra were obtained by electron impact $(70 \mathrm{eV})$ a scan rate of $0.5 \mathrm{~s}$.

\section{Methods}

NIR spectra (transflectance) were acquired by sampling at different places in a random order on the bottom of dishes. A wave number range of $4000-10000 \mathrm{~cm}^{-1}$ was used by applying $8 \mathrm{~cm}^{-1}$ resolution. Each spectrum was obtained from the mean of 16 different scans, and 15 NIR spectra were acquired for each dish. The 30 spectra were compared by chemometric statistical processing using dedicated software and a cluster analysis-type qualitative calibration model (NIRCAL 5.0), based on principal component analysis (PCA) theory.

One-third of the samples were randomly chosen from the software to develop calibrations (C-Set), and the remaining samples were used for validation (V-Set).

GC analysis of triglycerides was carried out by a method derived from EC Regulation No. 213 of 9 January 2001 (European Community 2001) provided for butter. The optimized method was previously published (Bononi et al. 2001), and its repeatability has been studied (Tateo and Bononi 2003).

\section{Results and discussion}

\section{Application of NIR technique}

The aforementioned method based on the NIR technique was designed to detect easily the presence of organic matter at the bottom of aluminium foil containers. This technique allowed a preliminary qualitative check (i.e. presence/absence) to be performed, and can be conveniently applied for quality control.

Figure 1 shows derivative normalized NIR spectra recorded over the wave number range 4200$4500 \mathrm{~cm}^{-1}$, where the most remarkable differences were observed; data were referred to one of the samples listed in Table I, namely sample P84/3. Group A spectra correspond to recordings carried out on the control dish (i.e. pure hexane evaporation), and group B spectra correspond to recordings

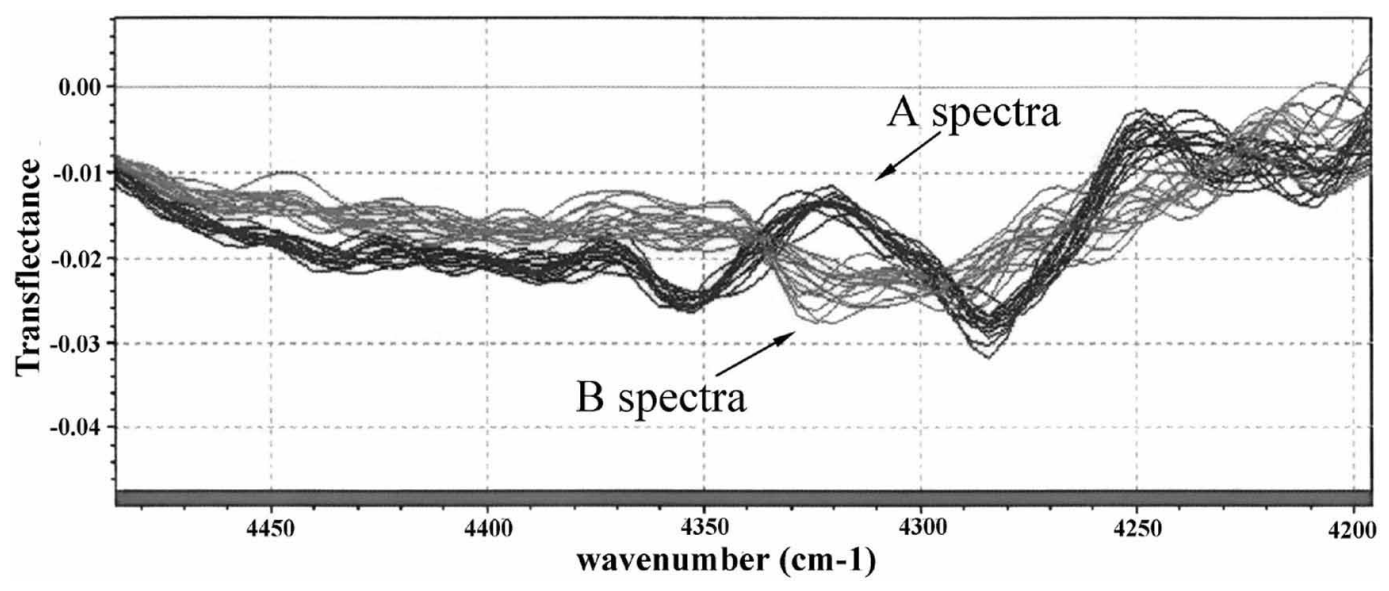

Figure 1. Example of first derivative NIR normalised spectra (sample P84/3). Group A spectra correspond to recordings carried out on the control dish (i.e. pure hexane evaporation). Group B spectra correspond to recordings carried out on dishes containing residue derived from vessel washing with hexane. 
Table I. Per cent characterization of triglycerides used as lubricants in aluminium foil vessels for household use available on the Italian market. Samples dimensions are 21-32 $14-22 \mathrm{~cm}$. Samples P84/2, P84/4 and P84/12 are round-shaped (diameter $=27 \mathrm{~cm}$ approximately.).

\begin{tabular}{|c|c|c|c|c|c|c|c|c|c|c|c|c|c|}
\hline Sample code & & P84/1 & $\mathrm{P} 84 / 2$ & P84/3 & P84/4 & P84/5 & P84/6 & P84/7 & P84/8 & P84/9 & P84/10 & $\mathrm{P} 84 / 11$ & P84/12 \\
\hline \multirow[t]{4}{*}{ Group G1 } & C24 & 10.7 & 11.8 & 12.0 & 16 & 14.6 & 15.4 & 13.8 & 14.4 & 23.0 & 48.2 & 39.0 & 20.1 \\
\hline & $\mathrm{C} 26$ & 21.9 & 21.3 & 23.6 & 30.5 & 27.4 & 26.2 & 25.5 & 25.9 & 37.9 & 20.1 & 42.8 & 39.7 \\
\hline & $\mathrm{C} 28$ & 15.0 & 13.1 & 15.5 & 18.2 & 16.3 & 15.3 & 15.2 & 14.7 & 19.2 & 2.0 & 14.6 & 25.9 \\
\hline & C30 & 3.4 & 5.2 & 3.5 & 2.9 & 3.6 & 3.5 & 3.1 & 2.9 & 4.0 & $<0.4$ & 1.4 & 5.6 \\
\hline \multirow[t]{5}{*}{ Group G2 } & C40 & 2.8 & 2.9 & 3.7 & 3.4 & 4.2 & 3.9 & 4.4 & 4.2 & $<0.4$ & $<0.4$ & $<0.4$ & $<0.4$ \\
\hline & $\mathrm{C} 42$ & 7.7 & 6.9 & 9.2 & 9.8 & 10.7 & 9.6 & 10.9 & 10.1 & $<0.4$ & $<0.4$ & $<0.4$ & $<0.4$ \\
\hline & C44 & 7.8 & 6.5 & 8.9 & 8.9 & 9.5 & 8.3 & 9.6 & 8.5 & $<0.4$ & $<0.4$ & $<0.4$ & $<0.4$ \\
\hline & C46 & 3.7 & 3.4 & 3.9 & 3 & 3.7 & 3.5 & 3.8 & 3.1 & $<0.4$ & $<0.4$ & $<0.4$ & $<0.4$ \\
\hline & C48 & 1.0 & 1.1 & 0.6 & 0.4 & 0.5 & 0.5 & 0.5 & 0.7 & $<0.4$ & $<0.4$ & $<0.4$ & $<0.4$ \\
\hline \multirow[t]{9}{*}{ Group G3 } & C50 & 1.6 & 1.1 & 0.3 & $<0.4$ & $<0.4$ & 1.4 & $<0.4$ & 2.5 & $<0.4$ & $<0.4$ & $<0.4$ & $<0.4$ \\
\hline & C52 & 1.7 & 1.6 & 1.3 & $<0.4$ & $<0.4$ & 2.9 & 0.3 & 3.2 & 5.0 & $<0.4$ & $<0.4$ & $<0.4$ \\
\hline & C54 & 6.2 & 2.1 & 1.7 & $<0.4$ & $<0.4$ & 2.7 & 0.4 & 2.2 & 10.9 & $<0.4$ & $<0.4$ & $<0.4$ \\
\hline & C56 & 5.8 & 3.2 & 2.8 & 0.6 & 1.1 & 0.8 & 1.3 & 0.9 & $<0.4$ & $<0.4$ & $<0.4$ & $<0.4$ \\
\hline & C58 & 6.1 & 4.6 & 4.0 & 2.6 & 3.2 & 2.4 & 3.8 & 2.9 & $<0.4$ & $<0.4$ & $<0.4$ & $<0.4$ \\
\hline & C60 & 3.5 & 5.2 & 3.9 & 3 & 3.7 & 2.8 & 4.8 & 2.9 & $<0.4$ & $<0.4$ & $<0.4$ & $<0.4$ \\
\hline & C62 & 1.1 & 4.1 & 2.2 & 0.7 & 1.5 & 0.8 & 2.6 & 0.9 & $<0.4$ & $<0.4$ & $<0.4$ & $<0.4$ \\
\hline & Others & $<0.4$ & 5.8 & 3.1 & $<0.4$ & $<0.4$ & $<0.4$ & $<0.4$ & $<0.4$ & $<0.4$ & 29.7 & 2.2 & 8.7 \\
\hline & Total & 100 & 100 & 100 & 100 & 100 & 100 & 100 & 100 & 100 & 100 & 100 & 100 \\
\hline
\end{tabular}

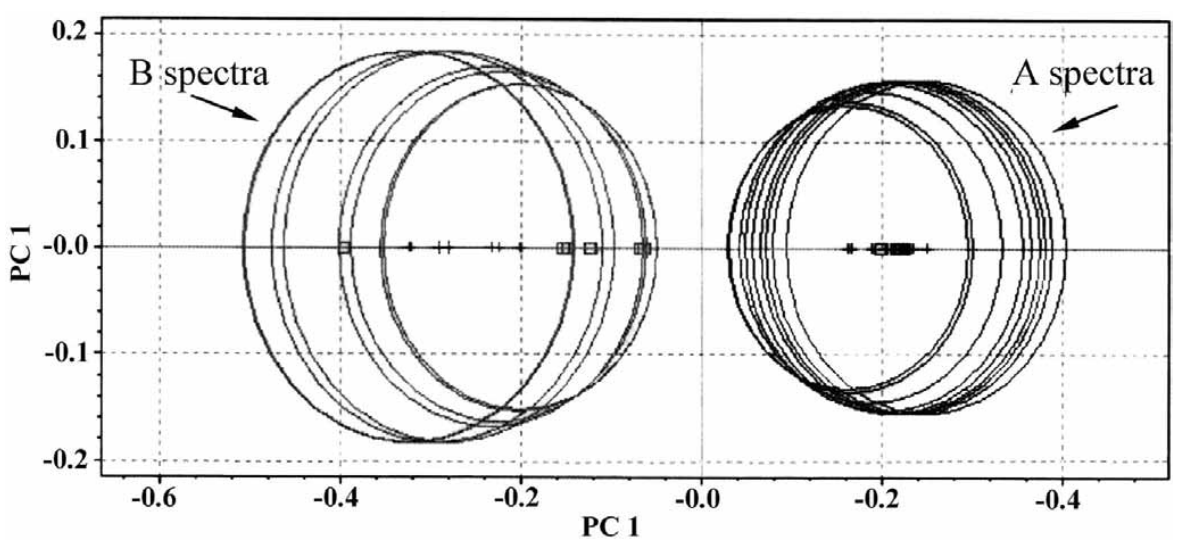

Figure 2. PCA plot, on the first principal component, for NIR spectra obtained from sample designated as P84/3. Cluster A was relevant to group A spectra (control), and cluster B to group B spectra (dishes containing extraction residue).

carried out on dishes containing the dried residue derived from vessel washing with hexane. Similar results were drawn for all other samples analysed.

Figure 2 shows a PCA plot on the first principal component, for spectra obtained from sampling. Ellipses correspond to plots for calibration samples (C-Set), and squares correspond to plots for validation (V-Set). Cluster A was relevant to group A spectra (reference), and cluster B to group B spectra (dishes containing extraction residue). FT-NIR analysis allowed for distinction of aluminium containers that contained 'contaminant' substances.

FT-IR analysis also proved useful for the detection of organic contaminants in the containers. Figure 3 shows a comparison example between three
IR spectra recorded between 4000 and $400 \mathrm{~cm}^{-1}$ for samples P84/2, P84/3, and P84/11. The presence of $\mathrm{C}=\mathrm{O}$ st $\left(1743 \mathrm{~cm}^{-1}, 1790-1650 \mathrm{~cm}^{-1}\right)$ and $\mathrm{C}-\mathrm{O}-\mathrm{C}$ st as $\left(1300-1000 \mathrm{~cm}^{-1}\right)$ signals was confirmed in addition to C-H st $\left(3000-2855 \mathrm{~cm}^{-1}\right)$ signals.

\section{Validation data for $G C$ analysis of triglycerides}

As stated in our previous work (Tateo and Bononi 2003) for the method used to calculate the relative per cent distribution of triglycerides, the precision was determined by means of relative standard deviations (RSD) of three series of six consecutive analyses, performed by three different operators, analysing the same certified butter triglyceride 


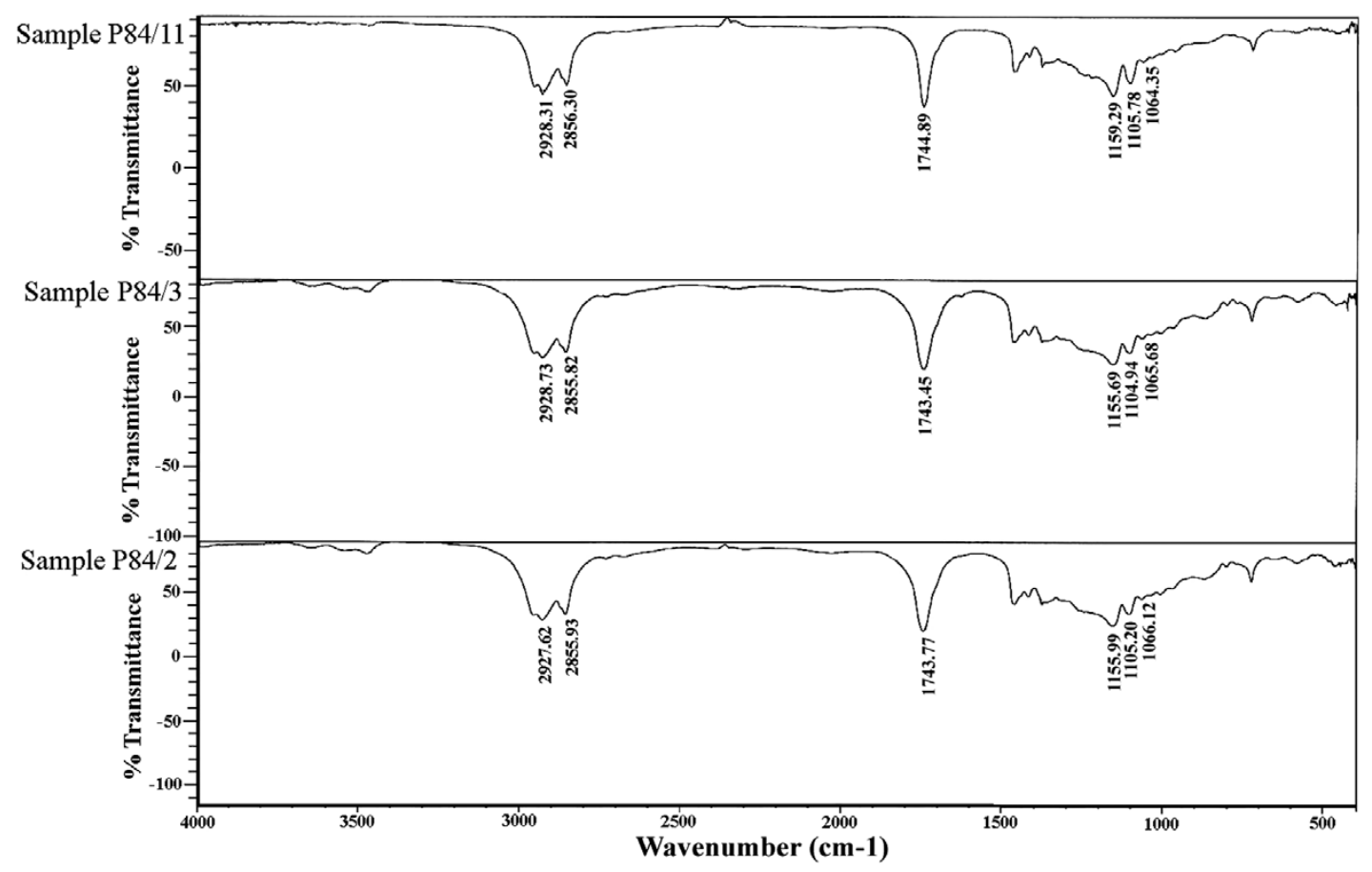

Figure 3. Comparison of FT-IR spectra of the investigated sample P84/3 together with those of sample P84/2 and P84/11.

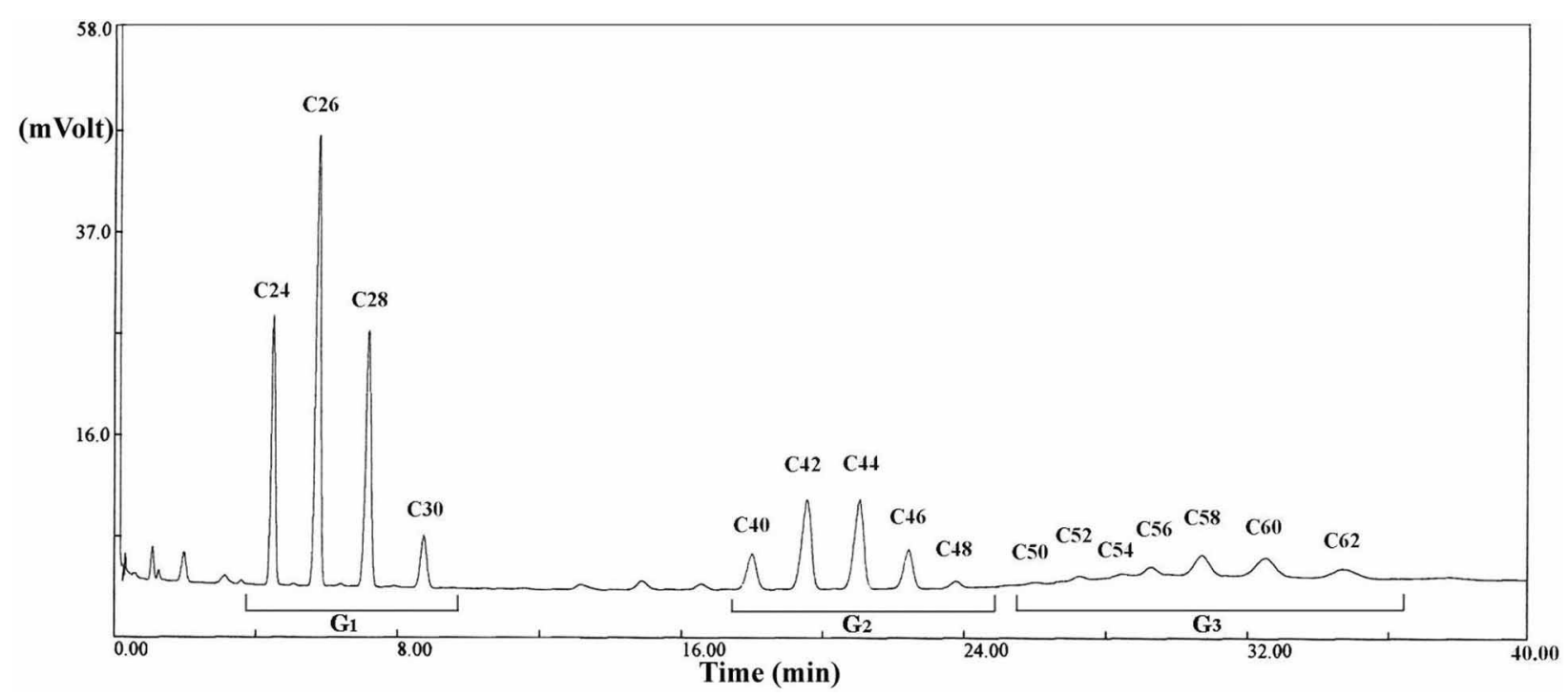

Figure 4. GC triglycerides profile, extracted from aluminium foil vessel (sample P84/3).

sample. The highest RSD value achieved was $4.06 \%$ for triglyceride C24 (one of the lowest represented components in the standard sample), $1.26 \%$ for triglyceride C34 (abundant on the average), and $0.71 \%$ for triglyceride C52 (predominant in the same standard sample).

In order to obtain adequate validation of the method used to show indicative significant data on contamination level, the internal standard triglyceride C21 (dissolved in hexane) was added to different containers for concentrations ranging from 5 to $360 \mu \mathrm{g} \mathrm{dm}^{-2}$. An RSD of ten consecutive analyses was 1.24 and $0.81 \%$ at the lowest and highest concentrations, respectively. The linear range was $10-500 \mu \mathrm{g} \mathrm{ml}^{-1}$ for triglyceride C21 hexane solution. Recovery values varied from 94 to $105 \%$ for reference (internal standard) spiking levels of 5 and $400 \mu \mathrm{g} \mathrm{dm}^{-2}$, respectively.

\section{$G C$ and $M S$ analysis results}

Table I shows results from analyses carried out on hexane extracts. Peak designation was that 

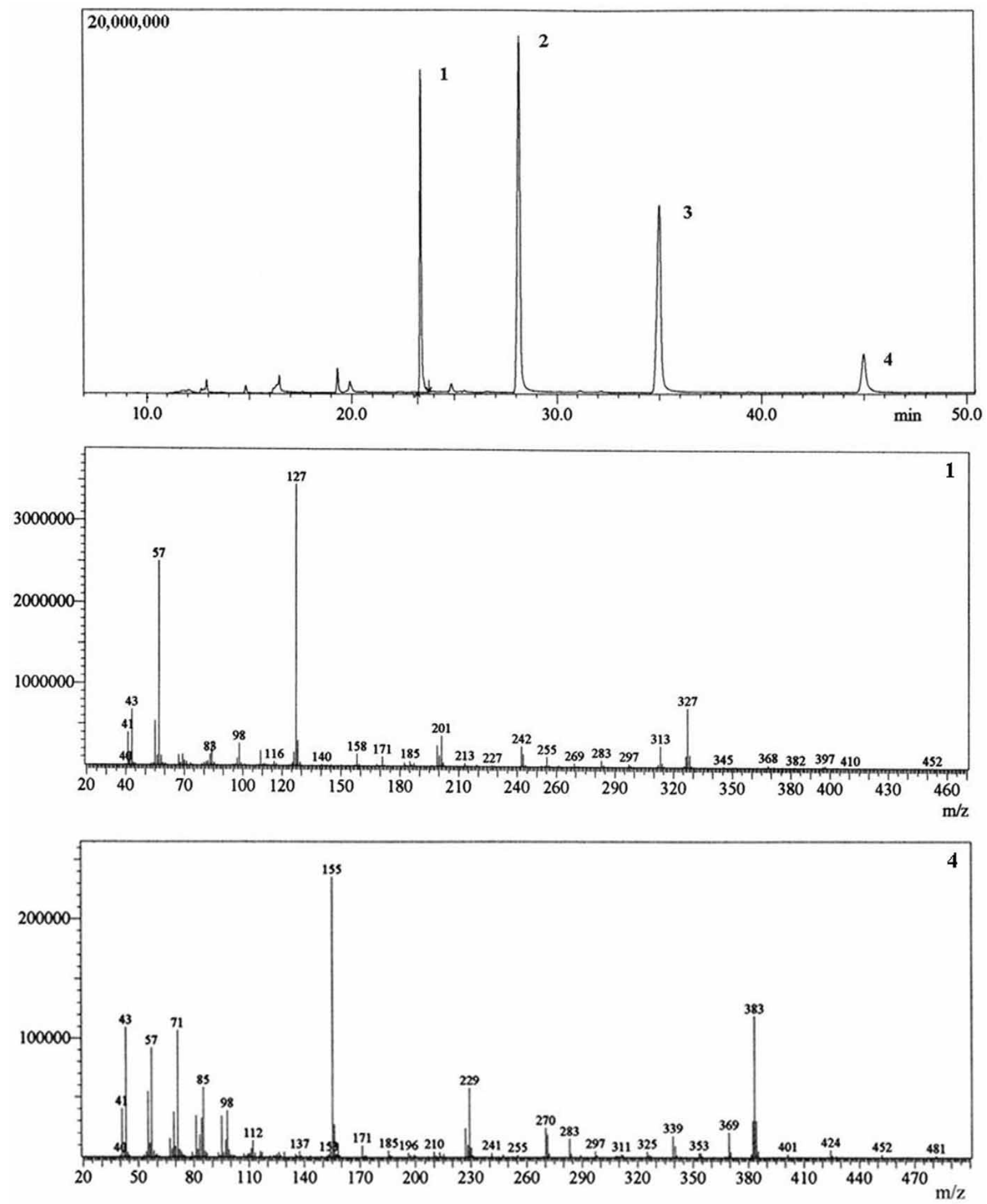

Figure 5. GC/MS trace (triglycerides group G1) produced by injection of hexane extract of organic substances (sample P84/5). Peaks are identified as follows: 1, Glycerol Tricaprylate; 2, triglycerides C26; 3, triglycerides C28; 4, Glycerol Tricaprate (sample P84/5).

conventionally used for analysis of triglycerides (Bononi et al. 2001). Results show substantially similar results for the different vessel samples, and three groups of triglycerides, which were classified as G1, G2, and G3, were found to be present. The trace corresponding to sample
P84/3 was reported as an example of gas chromatographic resolution in Figure 4. Based on relative percent values, it can be inferred that the three classes were synthesis glycerides, as the profile of peaks could not be assimilated to that of any natural fat matter. 
Correction factors for quantitative evaluation of individual triglycerides and other esters were assumed to be one. The total amount of contaminant substances resulted in a range of $897 \mu \mathrm{g} \mathrm{dm}^{-2}$ (sample P84/5) to $1209 \mu \mathrm{g} \mathrm{dm}^{-2}$ (sample P84/7).

Based on GC/MS analysis, peak C24 was identified as glycerol tricaprylate, C30 as glycerol tricaprate, and $\mathrm{C} 26$ and $\mathrm{C} 28$ as triglyceride isomers of fatty acids $\mathrm{C} 8$ and C10. Figure 5 shows the GC/MS trace (Group G1) for sample P84/5, and the identification of glycerol tricaprylate and glycerol caprate. Mass spectra of peaks classified as groups G2 and G3 revealed a nature of triglyceride isomers of higher molecular weight than those of G1.

In some aluminium foil containers, mixtures identified by GC/MS as butyl palmitate $[\mathrm{m} / \mathrm{z}: 56$ (100), 57 (50.4), 73 (25.6), 116 (11.2), 129 (18.8), 185 (4.8), 213 (5.2), 239 (18.8), 257 (27.6), 312 (5.6)] and butyl stearate [m/z: 56 (100), 57 (54.4), 73 (28.4), 101 (8.0), 116 (18.8), 129 (24.4), 185 (8.4), 241 (7.2), 267 (23.6), 285 (44.0), 340 (26.0)] were present at a greater relative percentage concentration than C24, C26, C28, and C30 (e.g. sample P84/10). In a recent investigation on another sample vessel, isobutyl palmitate and isobutyl stearate were found to be predominant (data not shown).

Based on the above experiments, it is evident that substances other than aluminium are leached to food from aluminium foil containers. However, the use of this substance mixture has not been regulated and is not included on any positive lists for aluminium containers.

\section{Sensory evaluation tests}

To verify that the contaminants listed were transferred to food in quantities large enough to cause deterioration in sensory characteristics, the following experiment was performed. Briefly, extra virgin olive oil (approximately $2 \mathrm{ml}$ ) was added to aluminium foil containers containing the 'contaminants', and the same quantity of extra virgin olive oil was added to containers, previously washed with hexane. Thermal treatment simulating home baking was carried out $\left(150^{\circ} \mathrm{C}, 45 \mathrm{~min}\right)$, and extra virgin olive oil was used as a valid food simulating substance.

Only changes in taste were observed in extra virgin olive oil added to aluminium foil containers containing contaminants, and these changes were not identified when performing the same experiment aluminium foil containers previously washed with hexane. Atypical taste and odour imparted to oil were described as 'pungent', 'rancid', 'metallic', 'unpleasant', and 'extraneous to the flavour of cooked olive oil'. Based on these results, the aluminium containers used in this investigation, did not comply with EC Regulation No. 1935/ 2004 (European Community 2004).

\section{Conclusions}

Analytical methods suggested in the present work may be useful when performing quality control of aluminium foil containers for household use. Proper control requires regulators to be informed of applicable studies and results. The use of aluminium foil containers, which contain residues of the types identified in this study, should be used under the appropriate regulation of substances applied for technological purposes, such as lubricants and release agents.

Moreover, data on mutagenic effects and carcinogenicity have been reported for glycerol tricaprylate (C24) (National Toxicology Program 1994; Pors and Fuhlendorff 2002; Carcinogenic Potency Database (CPP) 2006), and safety data concerning all triglycerides of fatty acids having a higher molecular weight than $\mathrm{C} 24$ are not available. In particular, Italian Law No. 1104 (1962) does not permit the use of either esterified or synthetic oil for food use.

Butyl palmitate and butyl stearate are commonly used as lubricants in metal working, as spreading and softening agents in plastics, textiles, cosmetics, and rubber industries, and as paint and ink additives. Their use for aluminium containers has not yet been regulated by law.

\section{References}

Bononi M, Fossati A, Lubian E, Tateo F, Fasan S. 2001. Caratterizzazione dei trigliceridi di sintesi con funzione di solvente/supporto in aroma burro. Rivista Italiana delle Sostanze Grasse 78:465-469.

Carcinogenic Potency Database (CPP). 2006. (Berkeley, CA: Lawrence Berkeley Laboratory) (available at: http://potency. berkeley.edu/chempages/TRICAPRYLIN.html) (accessed on 3 April 2006).

European Commission. 1990. Commission Directive EEC/90/ 128 of 23 February 1990 relating to plastics materials and articles intended to come into contact with foodstuffs. Official Journal of the European Communities L 349.

European Community. 2001. Regulation (EC) No. 213/2001 of the Commission of the European Communities of 9 January 2001 laying down detailed rules for the application of Council Regulation (EC) No. 1255/1999 as regards methods for the analysis and quality evaluation of milk and milk products and amending Regulations (EC) No. 2771/1999 and No. 2799/ 1999. Official Journal of the European Communities L 37.

European Community. 2004. Regulation (EC) No. 1935/2004 of the European Parliament and of the Council of 27 October 2004 on materials and articles intended to come into contact with food and repealing Directives 80/590/EEC and 89/109/ EEC. Official Journal of the European Communities L 338. 
Feliciani R, Denaro M, Giamberardini S, Maggio A, Milana MR. 2005. Alluminio a contatto alimentare: studi sperimentali e aspetti tecnici. Notiziario dell'Istituto Superiore della Sanità 18:11-15.

Gramiccioni L, Ingrao G, Milana MR, Santaroni P, Tomassi G. 1996. Aluminium levels in Italian diets and in selected foods from aluminium utensils. Food Additives and Contaminants 13:767-774.

Grob K, Artho A, Biedermann M, Caramaschi A. 1992. Batching oils on sisal bags used for packaging foods: analysis by couplet LC/GC. Journal of the Association of Official Analytical Chemists International 75:283-287.

Grob K, Artho A, Biedermann M, Egli J. 1991b. Food contamination by hydrocarbons from lubricating oils and release agents: determination by coupled LC-GC. Food Additives and Contaminants 8:437-446.

Grob K, Lanfranchi M, Egli J, Artho A. 1991a. Determination of food contamination by mineral oil from jute sacks using coupled LC-GC. Journal of the Association of Official Analytical Chemists 74:506-512.

Italian Law No. 1104. Legge No. 1104 del 24 Luglio 1962 riguardante il divieto di esterificazione degli olii di qualsiasi specie destinati ad uso commestibile. Gazzetta Ufficiale no. 201, 10/08/1962 (available at: http://www.infoleges.it/
BancheDati/PDF/PDF.aspx?database $=1 \&$ PageSize $=$ A $4 \&$ PageOrientation $=$ Portrait $\&$ Columns $=2 \& i d=104208$ ) (accessed 7 March 2006).

Jickells SM, Nichol J, Castle L. 1994. Migration of mineral hydrocarbons into foods. 6. Press lubricants used in food and beverage cans. Food Additives and Contaminants 11:595-604.

National Toxicology Program. 1994. Comparative toxicology studies of corn oil, safflower oil, and tricaprylin (CAS Nos. 8001-30-7, 8001-23-8, and 538-23-8) in male F344/N rats as vehicles for gavage. Technical Report Series No. 426 (Research Triangle Park, NC) (available at: http://ntp-server.niehs.nih. gov/index.cfm?objectid=0709D0C9-A575-BA2F-3069EBD44 EA674E2) (accessed 30 November 2005).

Pors J, Fuhlendorff R. 2002. Survey of chemical substances from tampons. Survey No. 12 (available at: http://www.mst.dk/ chemi/PDF/Tamp4UK.pdf) (accessed 30 November 2005).

Quality Management System. 2004. Quality Management System Certificate Det Norske Veritas, Cert. 14110-2004-AQ-MIL SINCERT (available at: http://www.dnv.it/certificazione/lista clienti/index.asp? Company $=\& Z I P=$ Milano $\&$ country $=$ Italy $\&$ Standard $=\&$ Accreditation $=$ All $\&$ IndustryCode $=$ All $\&$ Scope $=$ \&page $=31$ ) (accessed 7 March 2006).

Tateo F, Bononi M. 2003. Verifiche di ripetibilità nell'analisi GC dei trigliceridi. Industrie Alimentari 42:500-504. 